\title{
Input-to-State Stability of Lur'e Hyperbolic Distributed Complex-Valued Parameter Control Systems: LOI Approach
}

\author{
Zhixin Tai, ${ }^{1}$ Xingcheng Wang, ${ }^{2}$ Yan Shi, ${ }^{3}$ and Hamid Reza Karimi ${ }^{4}$ \\ ${ }^{1}$ Institute of Dynamics and Control, School of Astronautics and Aeronautics, Tsinghua University, Beijing 100084, China \\ ${ }^{2}$ Department of Automation, School of Information Science and Technology, Dalian Maritime University, Dalian 116026, China \\ ${ }^{3}$ School of Industrial Engineering, Tokai University, Kumamoto 862-8652, Japan \\ ${ }^{4}$ Department of Engineering, Faculty of Engineering and Science, University of Agder, 4898 Grimstad, Norway
}

Correspondence should be addressed to Xingcheng Wang; xcwangdalian@yeah.net

Received 3 September 2012; Revised 25 December 2012; Accepted 9 January 2013

Academic Editor: Xinzhu Meng

Copyright (C) 2013 Zhixin Tai et al. This is an open access article distributed under the Creative Commons Attribution License, which permits unrestricted use, distribution, and reproduction in any medium, provided the original work is properly cited.

In this work, input-to-state stability of Lur'e hyperbolic distributed complex-valued parameter control systems has been addressed. Using comparison principle, delay-dependent sufficient conditions for the input-to-state stability in complex Hilbert spaces are established in terms of linear operator inequalities. Finally, numerical computation illustrates our result.

\section{Introduction}

Up to now, the overwhelming majority of stability analysis and control theory concerning the distributed parameter systems are all limited to the case where distributed parameter is real valued $[1,2]$. In this work, complex-valued systems that appear in such fields as quantum mechanics [3] and neural network [4] have been, for the first time, extended to the case of distributed complex-valued parameter systems where delay-dependent sufficient conditions for the input-to-state stability in complex Hilbert spaces are established in terms of linear operator inequality.

In this work, two new crucial lemmas used in complex Hilbert spaces will be developed and thereby our main results are given with detailed illustrations.

\section{Preliminaries}

Quantum control system, one of the major study intensities of control system, is a typical complex-valued distributed parameter system as also complex-valued neural network. Owing to the significance of this type of distributed parameter system, in view of the typical nonlinearity of Lur'e control system, consider the following Lur'e hyperbolic distributed complex-valued parameter control systems:

$$
\Sigma_{0}:\left\{\begin{array}{l}
\dot{\xi}(t)=A \xi(t)+B \xi(t-h)+C u(t)+D \eta(t), \\
z(t)=M \xi(t)+N \xi(t-h)+R u(t), \\
\eta(t)=-\varphi(t, z(t))
\end{array}\right.
$$

with the Neumann boundary condition $w^{(i)}(0, t)=$ $w^{(i)}(\pi, t)=0(i=0,1)$ and the initial condition $w(x, t)=\phi(x, t), t \in[-h, 0]$ in complex Hilbert spaces

$$
\begin{aligned}
\mathscr{H}=\{w: w(x, t)=\phi(x, t), t \in[-h, 0], \\
\\
|w| \in W^{2,2}((0, \pi), \mathbb{R}) \text { s.t. } \\
\quad \text { boundary condition } w(0, t)=w(\pi, t)=0\},
\end{aligned}
$$

where $w(x, t)$ is the complex-valued state, $i$ is the imaginary unit, $a_{0}>0, a_{1}<0$, and

$$
\begin{gathered}
\xi(t):=\left[\begin{array}{l}
w(x, t) \\
w_{t}(x, t)
\end{array}\right], \quad u(t):=\left[\begin{array}{l}
u_{1}(x, t) \\
u_{2}(x, t)
\end{array}\right], \\
\eta(t):=\left[\begin{array}{l}
\eta_{1}(x, t) \\
\eta_{2}(x, t)
\end{array}\right], \quad A:=\left[\begin{array}{cc}
0 & 1 \\
a_{0} \nabla^{2}-i a_{1} \nabla & -\mu_{0}
\end{array}\right],
\end{gathered}
$$




$$
\begin{gathered}
B:=\left[\begin{array}{cc}
0 & 0 \\
-a_{2} & -\mu_{1}
\end{array}\right] \quad C:=\left[\begin{array}{cc}
0 & 0 \\
-i b_{1} & b_{2}
\end{array}\right], \\
D:=\left[\begin{array}{cc}
0 & 0 \\
c_{1} & c_{2}
\end{array}\right], \quad M=\left[\begin{array}{cc}
m_{1} & 0 \\
0 & m_{2}
\end{array}\right], \\
N=\left[\begin{array}{cc}
0 & 0 \\
n_{1} & n_{2}
\end{array}\right], \quad R=\left[\begin{array}{cc}
0 & 0 \\
r_{1} & r_{2}
\end{array}\right]
\end{gathered}
$$

and where $\varphi(t, z(t)): R \times H \rightarrow H$ is an abstract nonlinear function satisfying the following sector condition:

$$
\left\langle\varphi(t, z(t))-K_{1} z(t), \varphi(t, z(t))-K_{2} z(t)\right\rangle \leq 0
$$

with operators

$$
K_{1}:=\left[\begin{array}{cc}
k_{11} & k_{12} \\
p_{11} \nabla^{2}+p_{12} & k_{13}
\end{array}\right], \quad K_{2}:=\left[\begin{array}{cc}
k_{21} & k_{22} \\
p_{11} \nabla^{2}+p_{22} & k_{23}
\end{array}\right] \text {. }
$$

Before proceeding, we shall introduce some notations and definitions as follows.

The set of such controls that are measurable and locally essentially bounded in complex Hilbert spaces $\mathcal{U}$ with the supremum norm $\|u\|_{\text {sup }}:=\sup \{\|u(t)\|: t \geq-h\}<\infty$ is denoted by $\mathscr{L}_{\infty}$.

For each $\phi \in C([-h, 0], \mathscr{H})$ and $u \in \mathscr{L}_{\infty}$, we denote by $w(t, \phi, u)$ the solution trajectory of systems (1) with initial state $\phi$ and control input $u$.

Definition 1. A function $\gamma: \mathbf{R}_{+} \rightarrow \mathbf{R}_{+}$is said to be a class $\mathscr{K}$-function if it is continuous, zero at zero and strictly increasing. A function $\beta: \mathbf{R}_{+} \times \mathbf{R}_{+} \rightarrow \mathbf{R}_{+}$is said to be a class $\mathscr{K} \mathscr{L}$-function if for each fixed $t \geq 0$, the function $\beta(\cdot, t)$ is a class $\mathscr{K}$-function and for each fixed $s \geq 0$, the function $\beta(s, \cdot)$ is decreasing and $\beta(s, t) \rightarrow 0$ as $t \rightarrow \infty$.

In what follows, we will have a position to define the concept of input-to-state stability (ISS) in complex Hilbert spaces.

Definition 2. System (1) is called input-to-state stable (ISS) in complex Hilbert spaces if there exist a class $\mathscr{K} \mathscr{L}$-function $\beta$ : $\mathbf{R}_{+} \times \mathbf{R}_{+} \rightarrow \mathbf{R}_{+}$and a class $\mathscr{K}$-function $\gamma: \mathbf{R}_{+} \rightarrow \mathbf{R}_{+}$such that for any initial state $\phi \in C([-h, 0], \mathscr{H})$ and any bounded control input $u \in \mathscr{L}_{\infty}$, it holds that

$$
\|x(t, \phi, u)\| \leq \beta\left(\|\phi\|_{h}, t\right)+\gamma\left(\|u\|_{\text {sup }}\right),
$$

where $\|\phi\|_{h}:=\sup \{\|\phi(\theta)\|:-h \leq \theta \leq 0\}$.

As a key tool for developing the input-to-state stability in this work, some lemmas will be presented and proved as follows.

Lemma 3 (see [5]). The following inequality holds:

$$
\begin{aligned}
\langle w(x, t), i \nabla w(x, t)\rangle \leq \frac{1}{2}(\langle & w(x, t), w(x, t)\rangle \\
& \left.-\left\langle w(x, t), \nabla^{2} w(x, t)\right\rangle\right) .
\end{aligned}
$$

Lemma 4 (see [5]). The following inequality holds:

$$
\left\langle w(x, t), \nabla^{2} w(x, t)\right\rangle \leq-\frac{1}{2}\langle w(x, t), w(x, t)\rangle
$$

Lemma 5 (see comparison principle [6]). If the function $g(x, y)$ is continuous and satisfies a Lipschitz condition, then the implication

$$
\left.\begin{array}{rl}
D_{+} m(x) & \leq g(x, m(x)) \\
D_{+} u(x) & \geq g(x, u(x)) \\
m\left(x_{0}\right) & \leq u\left(x_{0}\right)
\end{array}\right\} \Longrightarrow m(x) \leq u(x) \quad \text { for } t \geq t_{0}
$$

is true for continuous functions $m(x)$ and $u(x)$.

In the sequel, we shall give our main results using Lemmas 3,4 , and 5 .

\section{Main Results}

Theorem 6. Given a scalar $\beta>0$, if there exist scalars $q_{01}, q_{02}, q_{03}>0, \quad p_{1}>0, \varepsilon>0$ and positive definite realvalued matrices $Q>0$ and $P_{1}>0$ such that the following LMIs hold:

$$
\begin{gathered}
-\left(a_{0}-h_{3}\right)-\frac{1}{2} a_{1}<0, \quad a_{0}>0, a_{1}<0, \\
q_{02}-q_{03} \beta>0 \\
2 p_{1} \beta+a_{1}\left(q_{02}-q_{03} \beta\right)>0 \\
\left(a_{0}-h_{3}\right)\left(q_{02}-q_{03} \beta\right)-p_{1} \beta>0 \\
\Gamma:=\left[\begin{array}{cc}
\frac{1}{2}\left(a_{0}-h_{3}\right) q_{03}+\frac{3}{4} a_{1} q_{03} & 0 \\
0 & 0
\end{array}\right]+\left[\begin{array}{ll}
q_{01} & q_{02} \\
q_{02} & q_{03}
\end{array}\right]>0,
\end{gathered}
$$

$$
\left\{\begin{array}{cc}
\widehat{Q} \widehat{A}+(\widehat{Q} \widehat{A})^{T}+Q \widehat{Q}\left(B-D K_{1 m} N\right) \\
* & -e^{-2 \beta h} Q \\
* & * \\
* & \\
\widehat{Q} D-\varepsilon M^{T}\left(K_{2 m}-K_{1 m}\right)^{T} & \widehat{Q}\left(C-D K_{1 m} R\right) \\
-\varepsilon N^{T}\left(K_{2 m}-K_{1 m}\right)^{T} & 0 \\
-2 \varepsilon I & -\varepsilon\left(K_{2 m}-K_{1 m}\right) R \\
* & -2 P_{1}
\end{array}\right]<0,
$$


where

$$
\begin{aligned}
\widehat{Q} & :=\left[\begin{array}{cc}
q_{01}+\frac{1}{2}\left(a_{0}-h_{3}\right) q_{03}+\frac{3}{4} a_{1} q_{03} & q_{02} \\
q_{02} & q_{03}
\end{array}\right], \\
\widehat{A} & :=\left[\begin{array}{cc}
\beta & 1 \\
-h_{1}-\frac{1}{2}\left(a_{0}-h_{3}\right)-\frac{3}{4} a_{1} & \beta-\mu_{0}-h_{2}
\end{array}\right], \\
K_{1 m} & :=\left[\begin{array}{ll}
k_{11} & k_{12} \\
p_{12} & k_{13}
\end{array}\right], \quad K_{2 m}:=\left[\begin{array}{ll}
k_{21} & k_{22} \\
p_{22} & k_{23}
\end{array}\right], \\
h_{1} & :=c_{1} k_{11} m_{1}+c_{2} p_{12} m_{1}, \\
h_{2} & :=c_{1} k_{12} m_{2}+c_{2} k_{13} m_{2}, \\
h_{3} & :=c_{2} p_{11} m_{1} \\
& >0 .
\end{aligned}
$$

Then system (1) is input-to-state stable with decay rate $\beta>0$.

Proof. Using the loop transformation technique [7], it comes to conclude that the absolute input-to-state stability of system (1) in the sector $\left[K_{1}, K_{2}\right]$ is equivalent to that of the following system:

$$
\begin{gathered}
\dot{\xi}(t)=\left(A-D K_{1} M\right) \xi(t)+\left(B-D K_{1} N\right) \xi(t-h) \\
+D \widetilde{\eta}(t)+\left(C-D K_{1} R\right) u(t), \\
z(t)=M \xi(t)+N \xi(t-h)+R u(t), \\
\widetilde{\eta}(t)=-\widetilde{\varphi}(t, z(t)), \\
\xi(t)=\phi(t), \quad \text { for } t \in[-h, 0]
\end{gathered}
$$

in the sector $\left[0, K_{2}-K_{1}\right]$, where abstract nonlinear function $\widetilde{\varphi}(t, z(t))$ satisfies

$$
\left\langle\widetilde{\varphi}(t, z(t)), \widetilde{\varphi}(t, z(t))-\left(K_{2}-K_{1}\right) z(t)\right\rangle \leq 0 .
$$

Choose the following Lyapunov-Krasovskii functional in complex Hilbert spaces:

$$
V(t)=\langle\xi(t), P \xi(t)\rangle+\int_{-h}^{0} e^{2 \beta \theta}\langle\xi(t+\theta), Q \xi(t+\theta)\rangle \mathrm{d} \theta .
$$

It follows from (18) that

$$
\begin{aligned}
\dot{V}(t)+ & 2 \beta V(t)-2\left\langle u(t), P_{1} u(t)\right\rangle-2 \varepsilon\langle\widetilde{\eta}(t), \widetilde{\eta}(t)\rangle \\
& -2 \varepsilon\left\langle\widetilde{\eta}(t),\left(K_{2}-K_{1}\right)(M \xi(t)+N \xi(t-h)+R u(t))\right\rangle \\
= & \langle\chi(t), \Xi \chi(t)\rangle,
\end{aligned}
$$

where

$$
\chi(t):=\left[\begin{array}{c}
\xi(t) \\
\xi(t-h) \\
\widetilde{\eta}(t) \\
u(t)
\end{array}\right]
$$

$\Xi:=$

$$
\begin{gathered}
{\left[\begin{array}{ccc}
\left(A-D K_{1} M+\beta I\right)^{*} P+ & P\left(A-D K_{1} M+\beta I\right)+Q \\
* & \\
* & \\
* & \\
& & \\
P\left(B-D K_{1} N\right) & P D-\varepsilon M^{*}\left(K_{2}-K_{1}\right)^{*} & P\left(C-D K_{1} R\right) \\
-e^{-2 \beta h} Q & -\varepsilon N^{*}\left(K_{2}-K_{1}\right)^{*} & 0 \\
* & -2 \varepsilon I & -\varepsilon\left(K_{2}-K_{1}\right) R \\
* & * & -2 P_{1}
\end{array}\right] .}
\end{gathered}
$$

Taking the operators

$$
P:=\left[\begin{array}{c}
q_{01}-\left(a_{0} q_{03}+p_{1}\right) \nabla^{2}+i a_{1} q_{03} \nabla+p_{1} \nabla^{2}+h_{3} q_{03} \nabla^{2} \\
q_{02} \\
q_{02} \\
Q>0
\end{array}\right.
$$

the proof is given in the following steps.

Step 1. To prove that operator $P$ is self-adjoint positive definite operator:

Using Lemmas 3 and 4 and inequality (10), we have that

$$
\begin{aligned}
\left\langle w(x, t),-\left(a_{0}-h_{3}\right) q_{03} \nabla^{2} w(x, t)\right\rangle & \\
& +\left\langle w(x, t), i a_{1} q_{03} \nabla w(x, t)\right\rangle \\
\geq & \left(-\left(a_{0}-h_{3}\right) q_{03}-\frac{a_{1} q_{03}}{2}\right)\left\langle w(x, t), \nabla^{2} w(x, t)\right\rangle \\
& +\frac{a_{1} q_{03}}{2}\langle w(x, t), w(x, t)\rangle \\
\geq & \left(-\frac{1}{2}\right)\left(-\left(a_{0}-h_{3}\right) q_{03}-\frac{a_{1} q_{03}}{2}\right)\langle w(x, t), w(x, t)\rangle \\
& +\frac{a_{1} q_{03}}{2}\langle w(x, t), w(x, t)\rangle
\end{aligned}
$$

from which it follows that

$$
\begin{aligned}
& \langle\xi(t), P \xi(t)\rangle \\
& \geq\left\langle\left[\begin{array}{c}
w(x, t) \\
w_{t}(x, t)
\end{array}\right],\left(\left[\begin{array}{cc}
\frac{1}{2}\left(a_{0}-h_{3}\right) q_{03}+\frac{3}{4} a_{1} q_{03} & 0 \\
0 & 0
\end{array}\right]\right.\right. \\
& \left.\left.+\left[\begin{array}{ll}
q_{01} & q_{02} \\
q_{02} & q_{03}
\end{array}\right]\right)\left[\begin{array}{c}
w(x, t) \\
w_{t}(x, t)
\end{array}\right]\right\rangle .
\end{aligned}
$$

In view of LMI (13), positive definiteness of operator $P$ is verified. 
Step 2. In view of Lemmas 3 and 4 and inequalities (11)-(12), direct computation can obtain that

$$
\begin{aligned}
& \left\langle w(x, t),\left(-i a_{1}\left(q_{02}-q_{03} \beta\right) \nabla+p_{1} \beta \nabla^{2}\right) w(x, t)\right\rangle \\
& =\left\langle w(x, t),\left(-i a_{1}\left(q_{02}-q_{03} \beta\right) \nabla\right) w(x, t)\right\rangle \\
& \quad+\left\langle w(x, t), p_{1} \beta \nabla^{2} w(x, t)\right\rangle \\
& \leq \frac{1}{2}\left(-a_{1}\left(q_{02}-q_{03} \beta\right)\langle w(x, t), w(x, t)\rangle\right. \\
& \left.\quad+\left(2 p_{1} \beta+a_{1}\left(q_{02}-q_{03} \beta\right)\right)\left\langle w(x, t), \nabla^{2} w(x, t)\right\rangle\right) \\
& \leq \frac{1}{2}\left(-p_{1} \beta-\frac{3}{2} a_{1}\left(q_{02}-q_{03} \beta\right)\right)\langle w(x, t), w(x, t)\rangle
\end{aligned}
$$

from which it is easy to obtain, in view of LMI (14), that

$$
\begin{aligned}
& \langle\chi(t), \Xi \chi(t)\rangle \leq \\
& \left\langle\chi(t),\left[\begin{array}{cc}
\widehat{Q} \widehat{A}+(\widehat{Q} \widehat{A})^{H}+Q & \widehat{Q}\left(B-D K_{1 m} N\right) \\
* & -e^{-2 \beta h} Q \\
* & * \\
* & *
\end{array}\right.\right. \\
& \left.\left.\begin{array}{cc}
\widehat{Q} D-\varepsilon M^{T}\left(K_{2 m}-K_{1 m}\right)^{T} & \widehat{Q}\left(C-D K_{1 m} R\right) \\
-\varepsilon N^{T}\left(K_{2 m}-K_{1 m}\right)^{T} & 0 \\
-2 \varepsilon I & -\varepsilon\left(K_{2 m}-K_{1 m}\right) R \\
* & -2 P_{1}
\end{array}\right] \chi(t)\right\rangle
\end{aligned}
$$

which implies that the inequality $\dot{V}(t)+2 \beta V(t)-$ $2\left\langle u(t), P_{1} u(t)\right\rangle \leq 0$ holds for any $\chi(t)$ satisfying (17) and hence along the solution trajectories of system (16), by virtue of Lemma 5, we have that

$$
\begin{aligned}
& \lambda_{\min }(\Gamma)\|w(x, t)\|^{2} \leq V(t) \leq e^{-2 \beta t} V(0) \\
& +\frac{1}{\beta} \sup _{t \geq 0}\left\langle u(t), P_{1} u(t)\right\rangle \\
& =e^{-2 \beta t} V(0)+\frac{1}{\beta} \lambda_{\max }\left(P_{1}\right) \sup _{t \geq 0}\|u(t)\|^{2} \\
& \leq\left(e^{-\beta t} \sqrt{V(0)}+\sqrt{\frac{\lambda_{\max }\left(P_{1}\right)}{\beta}} \sup _{t \geq 0}\|u(t)\|\right)^{2} .
\end{aligned}
$$

It follows from (27) that

$$
\|w(x, t)\| \leq \frac{1}{\sqrt{\lambda_{\text {min }}(\Gamma)}}\left(e^{-\beta t} \sqrt{V(0)}+\sqrt{\frac{\lambda_{\max }\left(P_{1}\right)}{\beta}}\|u\|_{\text {sup }}\right) .
$$

Remark 7. To illustrate the utility of stability criteria established in this paper, applying Theorem 6 to the Lur'e distributed complex-valued parameter control systems (1) with coefficients $a_{0}=30, a_{1}=-0.12, a_{2}=3, \mu_{0}=$ 20, $\mu_{1}=-0.2, C=\left[\begin{array}{cc}0 & 0 \\ -4 i & -2\end{array}\right], D=\left[\begin{array}{cc}0 & 0 \\ -0.3 & 0.8\end{array}\right], \quad M=$ $\left[\begin{array}{ll}1 & 0 \\ 0 & 2\end{array}\right], \quad N=\left[\begin{array}{cc}0 & 0 \\ 1.2 & 0.3\end{array}\right], \quad R=\left[\begin{array}{cc}0 & 0 \\ 0.7 & 0.4\end{array}\right], K_{1 m}=\left[\begin{array}{cc}1 & 0 \\ 0 & 0.2\end{array}\right], K_{2 m}=$ $\left[\begin{array}{ll}2 & 0 \\ 0 & 0.9\end{array}\right], p_{11}=1, h_{1}=-0.30, h_{2}=0.32$, and $h_{3}=0.80$ yields that system (1) is input-to-state stable with decay rate $\beta=0.30$ and maximum delay $h_{\max }=2.2421$.

\section{Acknowledgments}

This work was partially supported by the National Natural Science Foundation of China (60976071, 61174058, 60974052, 61134001, and 60974071), the National Key Basic Research Program, China (2012CB215202), and the 111 Project (B12018).

\section{References}

[1] Z. Tai and S. Lun, "Dissipativity for linear neutral distributed parameter systems: LOI approach," Applied Mathematics Letters, vol. 25, no. 2, pp. 115-119, 2012.

[2] Z. Tai and S. Lun, "Absolutely exponential stability of Lur'e distributed parameter control systems," Applied Mathematics Letters, vol. 25, no. 3, pp. 232-236, 2012.

[3] C.-D. Yang, "Stability and quantization of complex-valued nonlinear quantum systems," Chaos, Solitons and Fractals, vol. 42, no. 2, pp. 711-723, 2009.

[4] Y. N. Zhang, Z. Li, and K. Li, "Complex-valued Zhang neural network for online complex-valued time-varying matrix inversion," Applied Mathematics and Computation, vol. 217, no. 24, pp. 10066-10073, 2011.

[5] Z. Tai, "Exponential stability of non-linear hyperbolic distributed complex-valued parameter systems," Applied Mathematics Letters, vol. 25, no. 10, pp. 1404-1409, 2012.

[6] E. Hairer, S. P. Nørsett, and G. Wanner, Solving Ordinary Differential Equations I: Nonstiff Problems, Springer, Berlin, Germany, 1991.

[7] H. K. Khalil, Nonlinear Systems, Prentice Hall, Upper Saddle River, NJ, USA, 1996.

And hence from Definition 2, the proof is completed. 


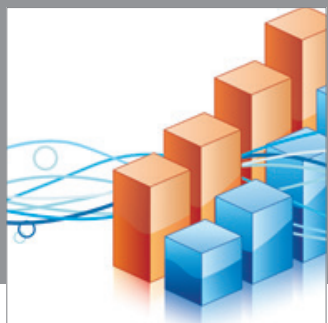

Advances in

Operations Research

mansans

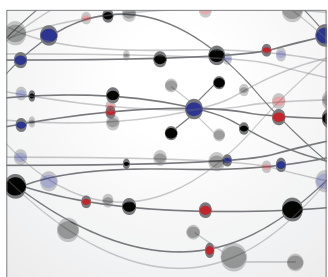

The Scientific World Journal
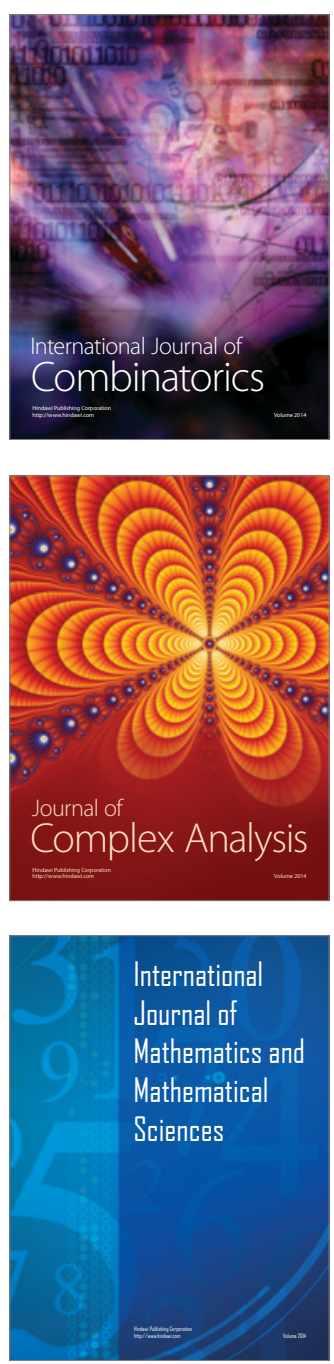
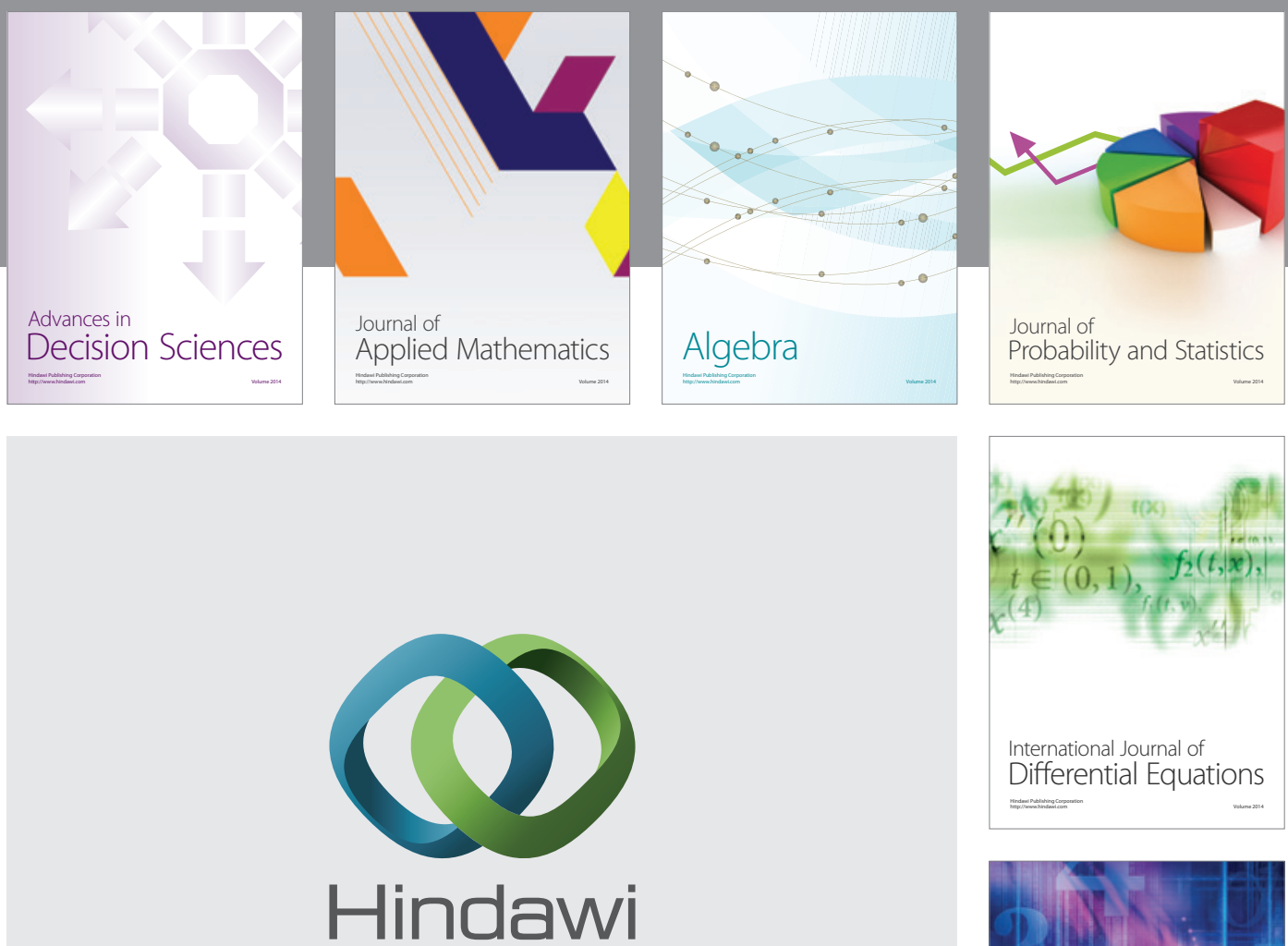

Submit your manuscripts at http://www.hindawi.com
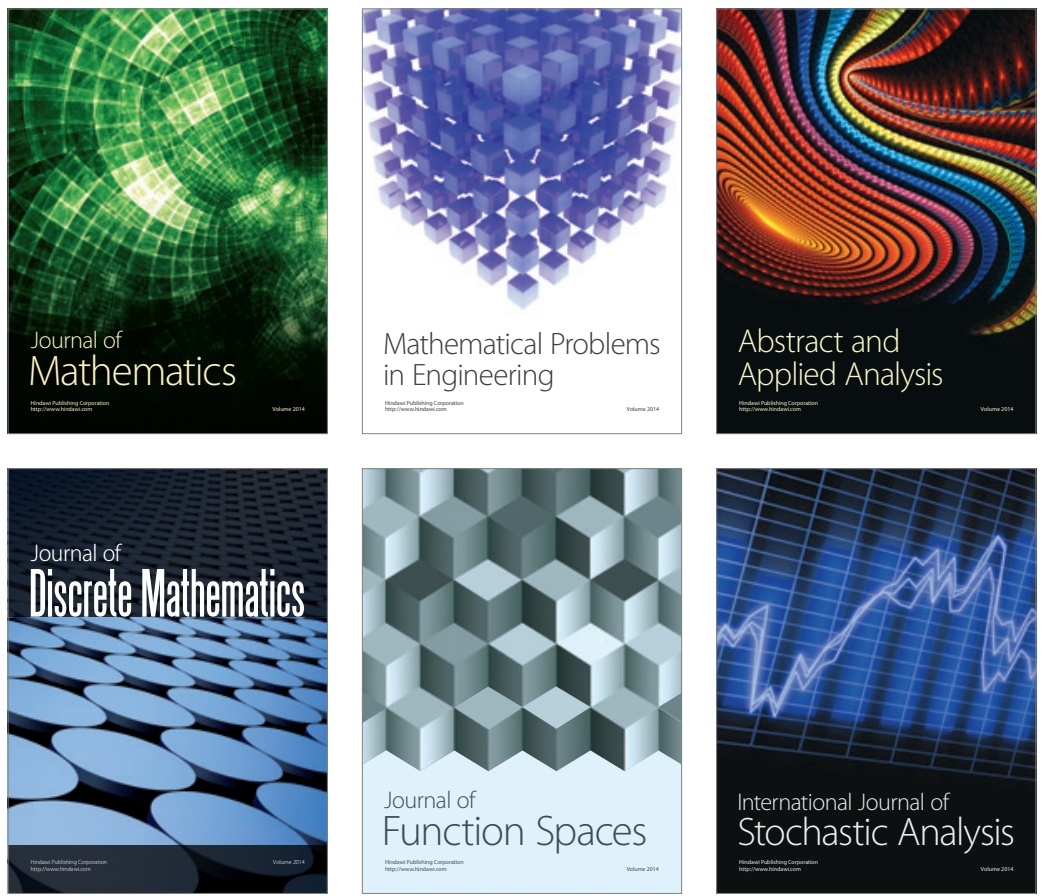

Journal of

Function Spaces

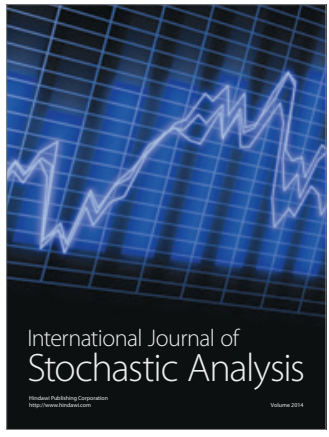

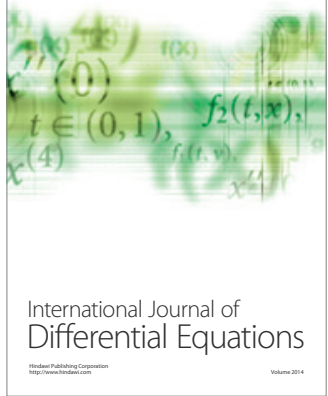
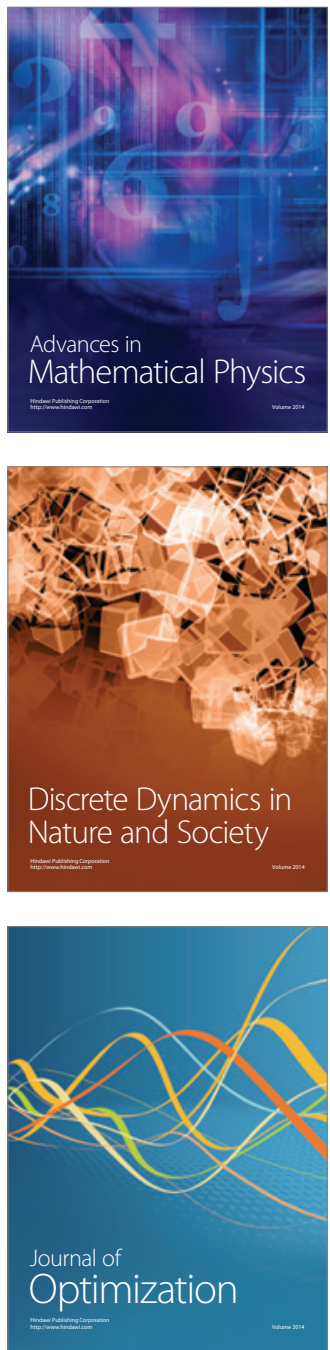